\title{
Long-Term Efficacy and Safety of Ultra Rapid Lispro (URLi) in Adults with Type 1 Diabetes: The PRONTO- T1D Extension
}

\author{
Juliana Bue-Valleskey • Leslie Klaff • Jang Ik Cho • Mary Anne Dellva • \\ Nanette C. Schloot · Janet Tobian · Junnosuke Miura • \\ Dominik Dahl
}

Received: October 7, 2020 / Accepted: December 15, 2020 / Published online: January 17, 2021

(C) The Author(s) 2021

\section{ABSTRACT}

Introduction: The PRONTO-T1D study, which evaluated the efficacy and safety of ultra rapid lispro (URLi) versus lispro in adults with type 1 diabetes (T1D), met the primary endpoint of noninferiority of HbA1c change from baseline compared to lispro at 26 weeks. We present results of an additional 26-week treatment phase evaluating long-term efficacy and safety of URLi.

Methods: In this phase 3, treat-to-target study, subjects were randomized to double-blind

Supplementary Information The online version contains supplementary material available at https:// doi.org/10.1007/s13300-020-00987-8.

J. Bue-Valleskey $(\bowtie) \cdot$ J. I. Cho $\cdot$ M. A. Dellva . J. Tobian

Eli Lilly and Company, Lilly Corporate Center, Indianapolis, IN, USA

e-mail: bue-valleskey_juliana_m@lilly.com

L. Klaff

Rainier Clinical Research Center, Renton, WA, USA

N. C. Schloot

Lilly Deutschland GmbH, Bad Homburg, Germany

J. Miura

Tokyo Women's Medical University School of

Medicine, Tokyo, Japan

D. Dahl

Gemeinschaftspraxis fur Innere Medizin und

Diabetologie, Hamburg, Germany mealtime URLi, lispro, or open-label postmeal URLi with insulin degludec or glargine for 26 weeks. Subjects in the double-blind URLi $(n=451)$ and lispro $(n=442)$ groups continued for another 26 weeks to assess long-term efficacy and safety.

Results: HbA1c increased marginally during the long-term maintenance period (week 26-52) in both groups to 7.47\% (URLi) and $7.54 \%$ (lispro). At week 52, there were no statistically significant treatment differences in change from baseline HbA1c with a leastsquares mean treatment difference (95\% confidence interval) of $-0.06 \%(-0.16,0.03)$. Proportions of patients with $\mathrm{HbA}_{1 \mathrm{c}}<7 \%$ at week 52 were similar (URLi, 26.8\%; lispro, $24.5 \%$ ). Self-monitored blood glucose (SMBG) showed that 1 -h $(9.23$ versus $10.14 \mathrm{mmol} / \mathrm{L})$ and 2 -h $(8.40$ versus $9.53 \mathrm{mmol} / \mathrm{L})$ postmeal daily mean glucose was statistically significantly $(p<0.001)$ lower with URLi than lispro. The rate and incidence of severe, documented, and postprandial hypoglycemia $(<54 \mathrm{mg} / \mathrm{dl}$ [3.0 mmol/L]) were similar between treatments, but URLi demonstrated a 31\% lower rate in the period more than $4 \mathrm{~h}$ after meals, $(p=0.023)$. Injection site reactions were reported by $3.3 \%$ of patients on URLi and $0.9 \%$ on lispro. The incidence of treatment-emergent adverse events was similar between treatments.

Conclusion: Overall glycemic control and improved postprandial glucose via SMBG were maintained after 52 weeks with URLi versus 
lispro, suggesting that the efficacy of URLi is preserved during long-term treatment in patients with T1D. No long-term safety issues were identified with URLi.

Trial Registration: ClinicalTrials.gov, NCT032 14367.

Keywords: Lispro; Type 1 diabetes; Ultra rapid lispro

\section{Key Summary Points}

\section{Why carry out this study?}

Ultra rapid lispro (URLi) is a novel insulin lispro formulation developed to more closely match physiological insulin secretion in response to meals and improve postprandial glucose control.

The PRONTO-T1D study, which evaluated the efficacy and safety of URLi versus lispro in adults with type 1 diabetes, met the primary endpoint of noninferior HbA1c change from baseline compared to lispro at 26 weeks, when insulins were dosed at mealtime in a double-blind manner.

An additional 26-week treatment phase for the double-blind mealtime treatment groups in PRONTO-T1D evaluated longterm efficacy and safety of URLi.

\section{What was learned from the study?}

Overall glycemic control and improved postprandial glucose via self-monitoring were maintained after 52 weeks of treatment with URLi versus lispro.

The efficacy and safety profile of URLi is preserved during long-term treatment in patients with type 1 diabetes.

\section{DIGITAL FEATURES}

This article is published with digital features, including a summary slide, to facilitate understanding of the article. To view digital features for this article go to https://doi.org/10.6084/ m9.figshare.13355963.

\section{INTRODUCTION}

Ultra rapid lispro (URLi) is a novel insulin lispro formulation developed to more closely match physiological insulin secretion in response to meals and improve postprandial glucose control [1]. The URLi formulation includes two key locally acting excipients, treprostinil and citrate, which accelerate the absorption of lispro from the site of injection via independent mechanisms of action [1]. Microdoses of treprostinil in URLi induce local vasodilation, while citrate increases vascular permeability $[2,3]$.

In a phase 1 , euglycemic clamp study comparing URLi to lispro (Humalog ${ }^{\circledR}$, Eli Lilly and Company) in patients with type 2 diabetes (T2D), URLi demonstrated earlier insulin action and a shorter duration of action [4]. In a fourperiod, crossover study in patients with type 1 diabetes (T1D), pharmacokinetics and postprandial glucose following a standardized test meal were characterized following administration of URLi, Humalog, NovoLog ${ }^{\circledR}$ (Novo Nordisk), and Fiasp ${ }^{\circledR}$ (Novo Nordisk) [1]. Patients received the same individualized, subcutaneous dose of each study insulin immediately prior to a liquid test meal. For comparison, 12 healthy subjects received the same test meal. URLi demonstrated the fastest insulin absorption and the greatest numeric postprandial glucose-lowering effect compared to the other insulins tested, and more closely matched the early physiological glucose control observed in healthy subjects [1]. URLi was efficacious with a similar safety profile to lispro (Humalog) in phase 3 studies of patients with T1D [5] or T2D [6].

The PRONTO-T1D study, which evaluated the efficacy and safety of URLi versus lispro in adults with $\mathrm{T} 1 \mathrm{D}$, met the primary endpoint of 
noninferior HbA1c change from baseline compared to lispro at 26 weeks, when insulins were dosed at mealtime in a double-blind manner [5]. Noninferiority for postmeal URLi (administered 20 min after the start of a meal, open-label) versus mealtime lispro was also shown [5]. Mealtime URLi was superior to mealtime lispro in controlling $1-\mathrm{h}$ and 2 -h postprandial glucose excursions during standardized test meals. Postmeal URLi provided similar postprandial glucose control during the test meal compared to mealtime lispro, but was less optimal compared to mealtime URLi. The results of the first 26 weeks of this trial have been published [5]. A continuous glucose monitoring substudy of PRONTO-T1D provided insight into ambient glucose in an unbiased, ad libitum feeding, ambulatory setting that offers additional evidence that URLi administered at the start of the meal provides superior postprandial glucose control compared to mealtime lispro. During the daytime period, URLi administered immediately before meals not only resulted in better postprandial glucose continuous glucose monitoring profiles but this also translated to more time spent in the target range of 3.9-$10.0 \mathrm{mmol} / \mathrm{L}(+43.6 \mathrm{~min}, p=0.020)$ with no increases in time spent in hypoglycemia compared to mealtime lispro, even though both groups reported similar HbA1c after 26 weeks of treatment [7].

An additional 26-week treatment phase for the double-blind mealtime treatment groups in PRONTO-T1D evaluated long-term efficacy and safety of URLi. This manuscript discusses the long-term efficacy and safety results from the 52-week treatment period for the two blinded treatment groups.

\section{METHODS}

\section{Study Design}

A detailed description of the study design and primary results has been published [5]. Briefly, this was a phase 3, treat-to-target study comparing URLi and lispro as part of a multiple daily injection regimen in adult patients with T1D (Fig.S1 in the supplementary material). Patients were treated with either insulin glargine or insulin degludec throughout the study in combination with prandial insulin. Following an 8-week, lead-in period for basal insulin optimization, patients were randomized to one of three groups and were permitted to use carbohydrate counting or pattern adjustment to manage prandial insulin dosing requirements. In two of the treatment groups, URLi and lispro were administered immediately (0-2 min) prior to each meal (mealtime) in a double-blind manner. A third open-label treatment group consisted of URLi administered 20 min after the start of a meal (postmeal URLi). In the openlabel treatment group, Japanese patients completed their study participation after the 52-week endpoint and safety follow-up. These results will be reported in a separate manuscript. All other patients in the open-label treatment group completed their study participation after the 26-week primary endpoint, the results of which have been published [5].

The study was designed to demonstrate noninferiority of URLi compared with lispro in change from baseline to week 26 in HbA1c, when URLi or lispro was administered at the start of the meal. Patients in the two blinded arms continued with double-blind treatment for an additional 26 weeks for a total of 52 weeks to allow comparison of long-term efficacy and safety. Study visits were scheduled to occur weekly during lead-in, weekly/biweekly during weeks $0-12$, every 4-6 weeks during weeks $12-26$, and every 6-7 weeks during weeks 26-52 (Fig. S1 in the supplementary material).

The study was conducted in accordance with the ethical principles of the Declaration of Helsinki and the International Conference on Harmonisation Guideline for Good Clinical Practice. The PRONTO-T1D study protocol was reviewed and approved by institutional ethics committees at each study center. All participants provided written informed consent. 


\section{Participants}

Adult patients with T1D, diagnosed on the basis of the World Health Organization criteria, and continuously using insulin for at least 1 year were eligible for participation if treated with a rapid-acting insulin analogue at least 90 days and basal insulin at least 30 days prior to screening, with an HbA1c of 7.0-9.5\% (53.0-$80.3 \mathrm{mmol} / \mathrm{mol}$ ) and body mass index no greater than $35 \mathrm{~kg} / \mathrm{m}^{2}$.

\section{Interventions and Randomization}

\section{Basal Dose Titration}

During the lead-in period, patients were treated with insulin glargine U100 (100 units $/ \mathrm{mL}$ ) once $(n=584 ; 47.8 \%)$ or twice daily $(n=99 ; 8.1 \%)$ or insulin degludec U100 once daily $(n=539$; $44.1 \%$ ) as determined by the investigator. The same basal insulin regimen (type, time of day, and frequency) was used throughout lead-in and treatment periods. Basal insulin dose was titrated to a fasting blood glucose (FBG) target of $5.6 \mathrm{mmol} / \mathrm{L}$ by the end of lead-in. Dose assessments were made at least weekly and adjustments every 3-4 days when appropriate. A detailed description of the basal insulin adjustment plan has been published [5].

\section{Bolus Dose Titration and Randomiza-} tion Patients were switched from their prestudy prandial insulin to lispro (unit for unit) at the start of lead-in. Prandial insulin doses were not changed during lead-in unless adjustments were necessary for safety reasons or to facilitate basal insulin optimization. After lead-in, patients were randomly assigned to mealtime URLi, mealtime lispro, or postmeal URLi in a $4: 4: 3$ randomization ratio.

Randomization was determined by a computer-generated random sequence using an interactive web-response system and stratified by country, baseline HbA1c $(\leq 7.5 \%,>7.5 \%)$, basal insulin type (glargine, degludec), and method of prandial insulin adjustment (carbohydrate counting or pattern adjustment).

During the initial 12 weeks of treatment, prandial insulin was adjusted as necessary to meet target self-monitored blood glucose (SMBG) levels in line with recommendations by the American Association of Clinical Endocrinologists $[5,8]$. During the maintenance period (weeks 12-26), prandial and basal insulin doses were only adjusted if necessary, to maintain glycemic control or for safety reasons. During the long-term maintenance period (weeks 26-52), investigators in discussion with patients titrated prandial and basal insulin as needed to maintain or optimize glycemic control. Recommended insulin titration algorithms were provided in the protocol and decisions to adjust insulin dose for each patient were the investigator's responsibility.

\section{Self-Monitored Blood Glucose}

Patients performed 10-point SMBG profiles prior to scheduled visits at premeal, $1-h$, and 2-h after the start of the morning, midday, and evening meals, and at bedtime. Patients were also instructed to perform daily measurements before morning, midday, and evening meals, bedtime, and as needed for glucose self-management. Patients were encouraged to perform SMBG whenever hypoglycemia was suspected and record blood glucose (BG) values and timing of events relative to meals. Patients were to treat BG less than $3.9 \mathrm{mmol} / \mathrm{L}$ as hypoglycemia.

\section{Statistical Methods}

Actual and change from baseline in HbA1c up to week 52 were analyzed using a mixed-effects model for repeated measurements (MMRM). The MMRM provides a consistent estimator when data are missing at random. The model implicitly adjusts for missing data through a variance-covariance structure. The MMRM included the fixed class effects of treatment, strata (pooled country, type of basal insulin, and prandial insulin dosing plan), and covariate of baseline HbA1c, visit, treatment-by-visit interaction, and an unstructured covariance structure. Additional continuous efficacy variables, as well as the change from baseline for these variables, were analyzed similarly with MMRM. 
Safety analyses were conducted on all randomized patients who received at least one dose of investigational product. Anti-insulin lispro antibodies were measured throughout the study. Patient-reported adverse events (including serious adverse events [SAEs] and treatmentemergent adverse events [TEAEs]) were summarized by preferred term and/or system organ class using the Medical Dictionary for Drug Regulatory Activities, version 21.1. When statistical comparisons were applied, Fisher's exact test was used. Severe hypoglycemia (an episode requiring assistance due to neurological impairment as confirmed by the investigator) was reported as an SAE. For other categories of hypoglycemia, rate and incidence of events were analyzed using a negative binomial regression model and a logistic regression model, respectively.

All statistical analyses were performed using SAS software, version 9.4 or higher (SAS Institute, Cary, NC, USA).

\section{RESULTS}

\section{Demographics and Disposition}

A total of 893 patients were randomized to mealtime URLi $(n=451)$ or lispro $(n=442)$. Overall, $92 \%$ of these patients completed 52 weeks of study treatment. Patient disposition for the double-blind treatment groups from enrollment to week 52 is presented in Fig. S2 in the supplementary material. Patient disposition data for the open-label treatment arm from enrollment to week 26 have been previously reported [5]. Demographic and baseline characteristics for the double-blind treatment groups were similar (Table S1 in the supplementary material). More than half of the patients were male $(56.7 \%)$, the mean age of patients was 44.3 years, and the mean duration of diabetes was 18.9 years.

\section{Efficacy}

\section{HbA1c}

Mean HbA1c decreased in both groups from $8.03 \%$ at screening to a baseline of $7.34 \%$ with mealtime URLi and $7.33 \%$ with lispro after the 8 -week lead-in period. By week 26, mean HbA1c had stabilized to $7.22 \%$ with mealtime URLi and 7.29\% with lispro (Fig. 1). HbA1c increased marginally during the long-term maintenance period (week 26-52) in both groups to $7.47 \%$ (URLi) and $7.54 \%$ (lispro). At week 52, there were no statistically significant treatment differences in change from baseline HbA1c with a least-squares mean treatment difference $(95 \%$ confidence interval) of $-0.06 \%(-0.16,0.03)$ (Fig. 1).

After 52 weeks of treatment, no significant differences were observed between mealtime URLi and mealtime lispro groups in the proportion of patients achieving HbA1c targets $<7 \%$ (26.8\% versus $24.5 \%$, respectively) and $\leq 6.5 \%$ ( $12.2 \%$ versus $11.3 \%$, respectively).

Self-Monitored Blood Glucose At week 52, the URLi versus lispro group had statistically significantly lower SMBG levels at morning 1-h postmeal (9.23 versus $10.14 \mathrm{mmol} / \mathrm{L}$, respectively; $p<0.001$ ), at 2 -h postmeal (8.40 versus $9.53 \mathrm{mmol} / \mathrm{L}$, respectively; $p<0.001)$, and at midday 1 -h postmeal (8.93 versus $9.87 \mathrm{mmol} / \mathrm{L}$, respectively; $p<0.001$ ), and statistically significantly higher at evening premeal (9.62 versus $9.11 \mathrm{mmol} / \mathrm{L}$, respectively; $p=0.032$ ) (Fig. 2). Similarly, mealtime URLi resulted in statistically significantly greater improvements from baseline in the daily mean glucose and daily mean 1-h and 2-h postprandial glucose values and excursions compared to lispro (Table 1).

\section{Insulin Dose}

There were no significant treatment differences in daily basal and total insulin dose at week 52 (Table S2 in the supplementary material). The ratio of prandial to total insulin dose at week 52 was approximately $52 \%$ and similar in each treatment group. 


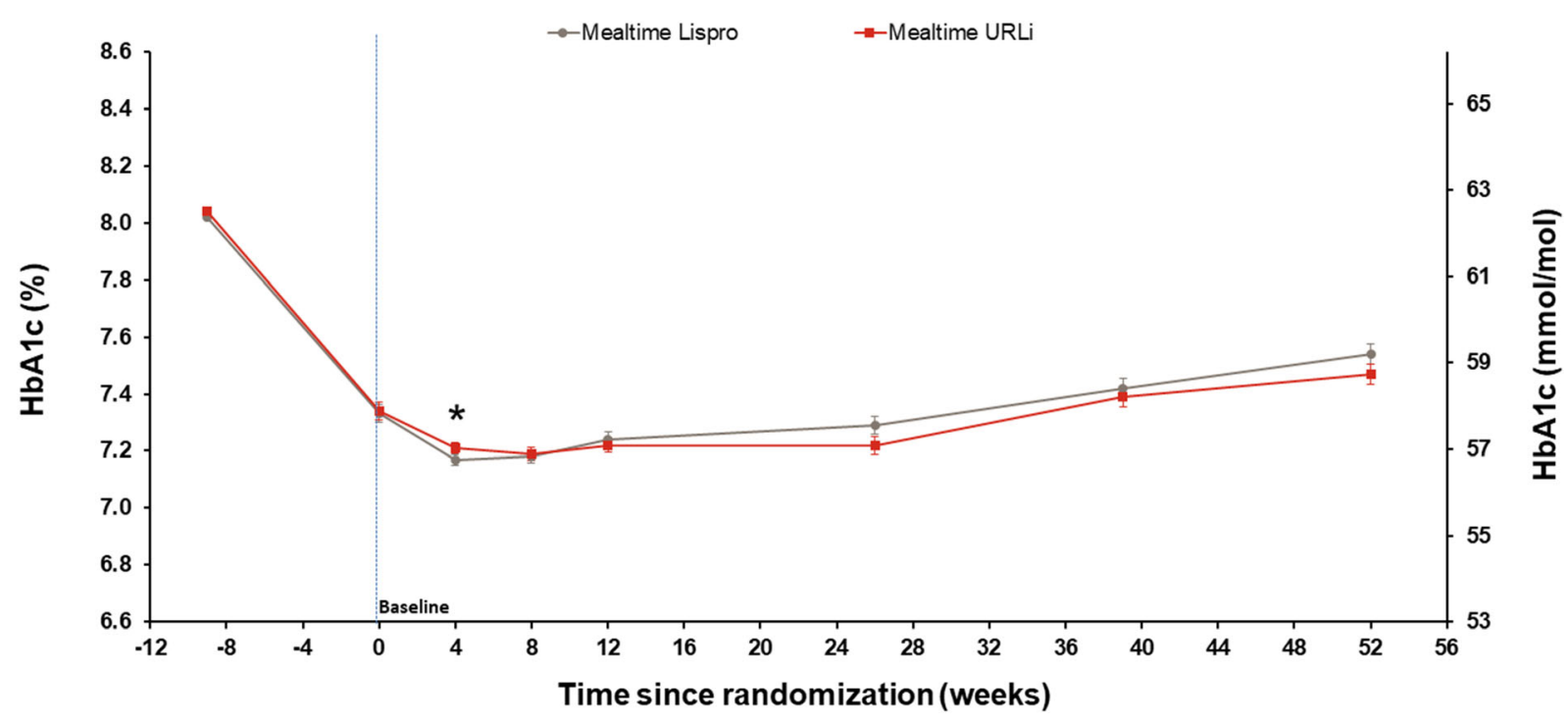

Fig. 1 Mean HbAlc from study entry to week 52. Data are mean at study entry and LSM (SE) at all other time points and based on the MMRM analysis. ${ }^{*} p<0.05$ for pairwise comparison of URLi versus lispro. LSM least- squares mean, MMRM mixed-effects model for repeated measures, SE standard error, URLi ultra rapid lispro

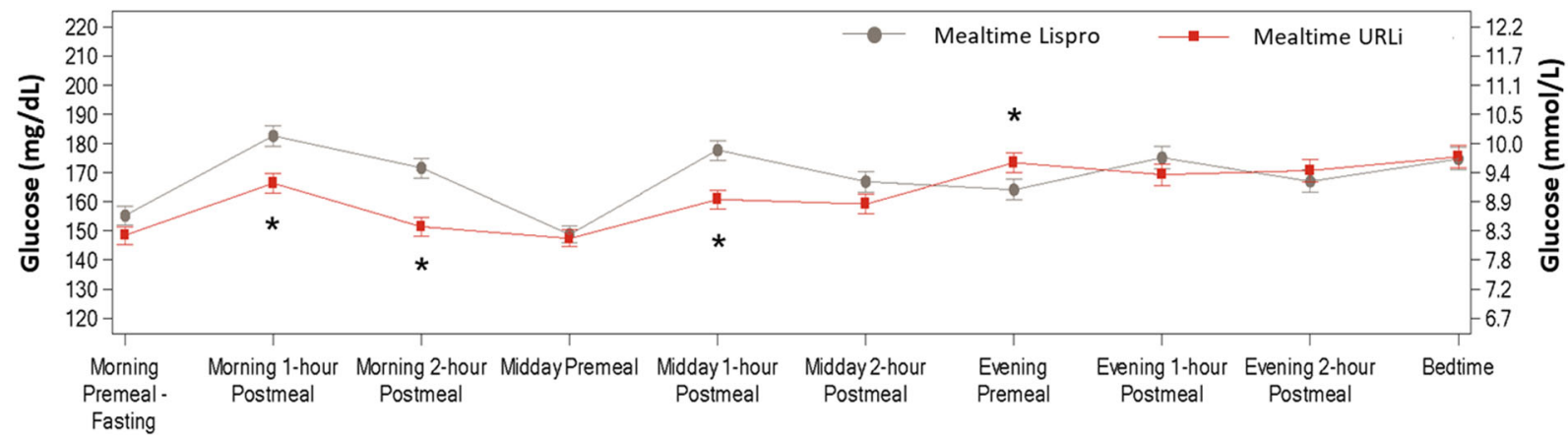

Fig. 2 Ten-point self-monitored blood glucose profile at week 52. Data are $\mathrm{LSM} \pm \mathrm{SE} .{ }^{*} p<0.05$ for pairwise comparison of mealtime URLi versus lispro. Data are

\section{Safety}

\section{Hypoglycemia}

The incidence and rate of severe hypoglycemia were similar between groups with 33 (7.3\%) patients reporting 53 episodes with mealtime URLi and 38 (8.6\%) patients reporting 67 episodes with lispro from baseline to week 52 . No significant differences were observed between groups in the rate or incidence of nocturnal and documented hypoglycemia (Fig. 3a). There were no clinically significant treatment differences in plotted as LSM (SE). LSM least-squares mean, SE standard error, URLi ultra rapid lispro

the rate and incidence of postprandial hypoglycemia; however, in the late postprandial period (more than $4 \mathrm{~h}$ after the meal), URLi was associated with a significant, $31 \%$ reduction in hypoglycemia compared to lispro $(p=0.023)$ (Fig. 3b).

\section{Adverse Events}

Two deaths occurred in the double-blind treatment groups from randomization to safety follow-up and were not considered related to study treatment: one patient in the mealtime URLi 
Table 1 Change from baseline to week 52 daily mean glucose and daily mean postprandial glucose levels and excursions from 10-point SMBG profile

\begin{tabular}{llllr}
\hline & Mealtime URLi & Mealtime Lispro & LSM difference (95\% CI) & $p$ value \\
\hline Daily mean glucose & & & & 0.009 \\
$\mathrm{mmol} / \mathrm{L}$ & $-0.29(0.10)$ & $0.04(0.11)$ & $-0.34(-0.59,-0.08)$ & \\
$\mathrm{mg} / \mathrm{dL}$ & $-5.3(1.9)$ & $0.8(1.9)$ & $-6.1(-10.6,-1.5)$ & $<0.001$ \\
$\begin{array}{l}\text { Daily mean 1-h PPG levels } \\
\mathrm{mmol} / \mathrm{L}\end{array}$ & $-0.56(0.13)$ & $0.19(0.14)$ & $-0.75(-1.08,-0.42)$ & \\
$\mathrm{mg} / \mathrm{dL}$ & $-10.1(2.4)$ & $3.4(2.5)$ & $-13.5(-19.4,-7.5)$ & \\
$\begin{array}{l}\text { Daily mean 2-h PPG levels } \\
\mathrm{mmol} / \mathrm{L}\end{array}$ & $-0.41(0.13)$ & $0.06(0.13)$ & $-0.46(-0.78,-0.15)$ & \\
$\mathrm{mg} / \mathrm{dL}$ & $-7.3(2.3)$ & $1.1(2.4)$ & $-8.4(-14.1,-2.7)$ & \\
$\begin{array}{l}\text { Daily mean 1-h PPG excursions } \\
\mathrm{mmol} / \mathrm{L}\end{array}$ & $-0.66(0.13)$ & $0.13(0.13)$ & $-0.79(-1.11,-0.48)$ & \\
$\mathrm{mg} / \mathrm{dL}$ & $-11.9(2.3)$ & $2.4(2.4)$ & $-14.3(-20.0,-8.6)$ & \\
$\begin{array}{l}\text { Daily mean 2-h PPG excursions } \\
\text { mmol/L }\end{array}$ & $-0.37(0.14)$ & $0.08(0.14)$ & $-0.46(-0.80,-0.11)$ & \\
$\mathrm{mg} / \mathrm{dL}$ & $-6.7(2.5)$ & $1.5(2.6)$ & $-8.2(-14.5,-1.9)$ & \\
\hline
\end{tabular}

Data are LSM (SE)

$C I$ confidence interval, $L S M$ least-squares mean, $P P G$ postprandial glucose, $S E$ standard error, $S M B G$ self-monitored blood glucose, $U R L i$ ultra rapid lispro

group (colon cancer); one in the lispro group (aortic stenosis). The incidences of SAEs, discontinuations from the study due to an $\mathrm{AE}$, and TEAEs were similar across groups (Table 2). A total of 122 patients (13.7\%) experienced at least one SAE. Severe hypoglycemia (requiring assistance due to neurological impairment, confirmed by investigator and reported as an SAE per protocol) was the most frequently reported SAE. The numbers of patients who experienced an $\mathrm{AE}$ leading to treatment discontinuation were similar between groups (and included four cases of pregnancy that were reported as AEs for tracking purposes, Table 2). Overall, 19 patients (2.1\%) experienced at least one injection site reaction TEAE, with a total of 31 injection site reaction TEAEs documented; all were of mild $(n=28)$ or moderate $(n=3)$ severity, and none resulted in discontinuation from study treatment. More patients in the mealtime URLi $(n=15 ; 3.3 \%)$ versus the lispro group $(n=4 ; 0.9 \%)$ experienced at least one injection site reaction TEAE, the most common being "injection site reaction" and "injection site pain". Six mild events were reported during the 26- to 52-week period; three per treatment group.

\section{Laboratory Assessments}

There were no clinically meaningful changes in any laboratory values from baseline to week 52 in either treatment group. Overall, no significant treatment differences were observed in the number of patients with treatment-emergent anti-insulin lispro antibodies. The mean percentage antibody binding values were low throughout the study and no between-treatment differences were noted after 4 weeks of 

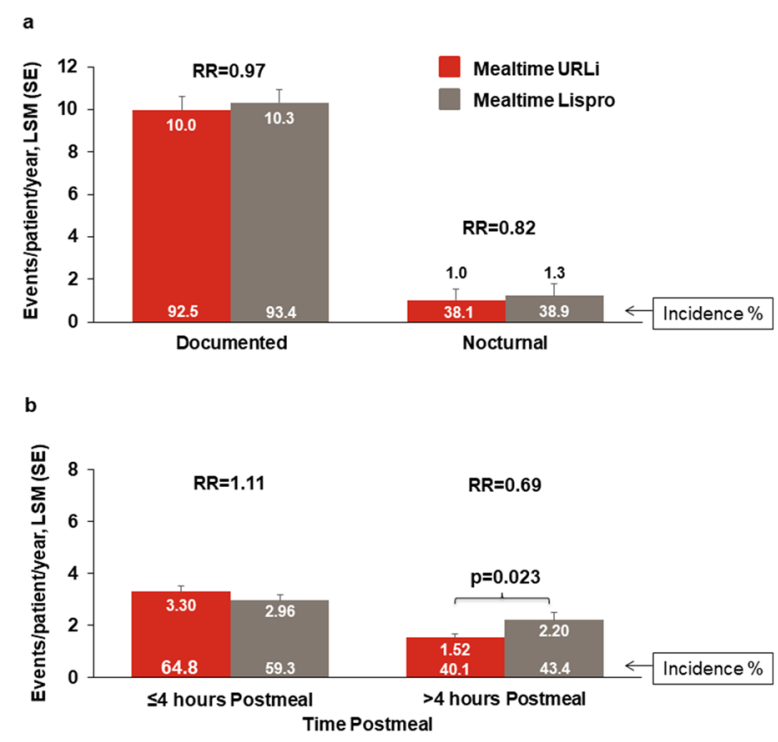

Fig. 3 Rate and incidence of hypoglycemia (with or without symptoms) from week 0 to week 52 (blood glucose less than $54 \mathrm{mg} / \mathrm{dL}[3.0 \mathrm{mmol} / \mathrm{L}])$. a Rate and incidence of documented and nocturnal hypoglycemia. b Rate and incidence of postmeal hypoglycemia. Data are LSM + SE for event rate and LSM for incidence. Nocturnal hypoglycemia was defined as documented hypoglycemia occurring between bedtime and waking. LSM, least squares mean, RR relative rate, SE standard error, URLi ultra rapid lispro

treatment (Fig. S3 in the supplementary material). No significant treatment differences were observed in change from baseline to week 52 for blood pressure, pulse, or body mass index. Average weight gain from baseline to week 52 was also similar between groups: $0.8 \mathrm{~kg}$ mealtime URLi; $0.9 \mathrm{~kg}$ lispro.

\section{DISCUSSION}

In this additional, 26-week treatment phase for the double-blind mealtime treatment groups in PRONTO-T1D, we evaluated the long-term efficacy and safety of URLi over 52 weeks of treatment in total. The findings are consistent with the initial 26-week data. Compared to mealtime lispro, treatment with URLi resulted in similar long-term HbA1c control and improved postprandial glucose control with similar daily insulin doses. The long-term safety profile of
URLi was similar to lispro. Compared to lispro, URLi was associated with no increase in severe, documented, or postprandial hypoglycemia risk and less late postprandial hypoglycemia. The overall adverse event profile was similar between treatments.

During each period of the study, HbA1c was similar in the double-blind treatment groups: HbA1c improved similarly during the lead-in and intensive titration period (week 0-week 12), remained stable during the maintenance period (week 12-week 26), and increased marginally during the long-term maintenance period (week 26-week 52). At week 52, there were no statistically significant treatment differences in actual or change from baseline HbA1c. The estimated treatment difference (URLi-lispro) at week 52 was similar to that previously reported at 26 weeks [5]. The proportions of patients meeting HbA1c targets were also similar between treatments throughout the study.

Compared to the mealtime lispro group, patients in the URLi group had lower postprandial blood glucose following breakfast and midday meals and lower daily mean glucose and glucose excursions by SMBG. These findings are consistent with the SMBG and mixed meal tolerance test results at 26 weeks [5] which showed superior postprandial glucose control of URLi compared to lispro.

The differences observed in $\mathrm{HbA} 1 \mathrm{c}$ reduction and postprandial glucose excursions were not due to differences in prandial, basal, or total daily insulin dosing as insulin dose (basal, bolus, and total) increased only marginally from baseline to week 52 and was similar at baseline and week 52 in both treatment groups. The ratio of prandial to total insulin dose at week 52 was also similar in both treatment groups (ca. $52 \%)$, consistent with the results at week 26 [5] and also with prior treat-to-target, multiple daily injection regimen studies in patients with T1D treated with insulin glargine and a rapidacting insulin analogue $[9,10]$.

The improvement in postprandial glycemic control with URLi compared to lispro control occurred without increasing the risk of severe, overall, nocturnal, or postprandial hypoglycemia. Postprandial hypoglycemia (documented BG less than $54 \mathrm{mg} / \mathrm{dl}$ [3.0 mmol/L] 
Table 2 Summary of adverse events over 52 weeks

\begin{tabular}{|c|c|c|}
\hline & $\begin{array}{l}\text { Mealtime URLi } \\
(N=451) \\
n(\%)\end{array}$ & $\begin{array}{l}\text { Mealtime Lispro } \\
(N=442) \\
n(\%)\end{array}$ \\
\hline Deaths $^{a}$ & $1(0.2)$ & $1(0.2)$ \\
\hline Serious adverse events & $54(12.0)$ & $68(15.4)$ \\
\hline Discontinuations from study due to an adverse event & $5(1.1)$ & $2(0.5)$ \\
\hline Discontinuations from study treatment due to an adverse event & $7(1.6)$ & $5(1.1)$ \\
\hline Treatment-emergent adverse events & $306(67.8)$ & $298(67.4)$ \\
\hline Combined eye disorder terms ${ }^{b}$ & $26(5.8)$ & $12(2.7)$ \\
\hline Combined injection site reaction terms ${ }^{c}$ & $15(3.3)$ & $4(0.9)$ \\
\hline Injection site reaction & $7(1.6)$ & $1(0.2)$ \\
\hline Injection site pain & $5(1.1)$ & 0 \\
\hline Lipohypertrophy & $1(0.2)$ & $3(0.7)$ \\
\hline
\end{tabular}

Subjects could be counted in more than one category

$N$ number of subjects in analysis population, $n$ number of subjects with at least one adverse event per event type, TEAE treatment-emergent adverse event, URLi ultra rapid lispro

${ }^{a}$ Deaths are also included as serious adverse events and discontinuation from study due to adverse events

b There was an imbalance in the incidence of patients reporting TEAEs in the system organ class "eye disorders"; however, no significant differences in individual preferred terms were observed

${ }^{c}$ Events not shown: injection site bruising, induration, and rash were reported once by URLi-treated patients and acquired lipodystrophy reported once by a lispro-treated patient

with or without symptoms) during the late postprandial period (more than $4 \mathrm{~h}$ after meals) was approximately $31 \%$ lower with mealtime URLi compared with lispro. A similar lowering of postprandial hypoglycemia was also observed at the week 26 primary endpoint [5]. These findings likely reflect the lower late insulin exposure and shorter duration of insulin exposure with URLi in comparison with lispro and other rapid insulin analogues as demonstrated in clinical pharmacology studies [1, 11].

URLi was well tolerated in this 52-week study. The safety profile and overall frequency of SAEs and discontinuations from the study due to AEs were similar between the URLi and lispro groups. As expected in this population, severe hypoglycemia was the most frequently reported SAE and there were no significant differences in the incidence or rate between groups.
Although the overall incidence was low, more injection site reaction TEAEs were reported with URLi compared to lispro over 52 weeks of treatment, although three mild events were reported for lispro and three mild events were reported for URLi after 26 weeks. The most common TEAE reported was injection site reaction; all events were reported as mild to moderate in severity, and no patients discontinued treatment because of an injection site reaction. This imbalance is consistent with combined injection site reaction TEAEs reported up to week 26 of treatment [5] and results from a multiple daily injection therapy with URLi in T2D [6] as well as with the overall incidence reported for other approved insulins [12, 13]. The exact cause of the increase in local injection site symptoms is unknown but could be attributable to one or both enabling excipients in the URLi formulation (citrate and 
treprostinil). Anti-insulin lispro antibody levels were low and no clinically meaningful differences in treatment-emergent anti-insulin lispro antibodies were observed. Similarly, there were no treatment differences in the incidence of patients experiencing potential systemic hypersensitivity TEAEs, with one potential systemic hypersensitivity reaction reported as related to study drug in each treatment group.

Strengths of this study include the doubleblind design, high completion rate (92\%), and the global nature of the study. It should be noted that many of the observations reported here were included as key secondary objectives in the protocol but may not have been adequately powered, and multiplicity adjustments were not performed. Although still more frequent than standard of care, one potential limitation may be the lower visit frequency in the second 26-week phase of the study, which may have contributed to the gradual decline in glycemic control in both treatment groups. In addition, as continuous glucose monitoring use for management of insulin therapy becomes more common, its use throughout the study as a research tool would also have added to understanding of time in target range and time below range beyond what was possible with conventional SMBG monitoring as performed in the current study. Nonetheless, results from the continuous glucose monitoring substudy performed as part of the 26-week period [7] support observations related to hypoglycemia risk reported here for the 52-week treatment period.

\section{CONCLUSIONS}

Overall glycemic control and improved postprandial glucose via self-monitoring were maintained after 52 weeks of treatment with URLi versus lispro. These observations suggest that the efficacy and safety profile of URLi is preserved during long-term treatment in patients with T1D.

\section{ACKNOWLEDGEMENTS}

Funding. This study and the Rapid Service Fee for this publication were funded by Eli Lilly and Company.

Medical Writing Assistance. The authors thank Michelle Carey, PhD, of Syneos Health for providing writing assistance funded by Eli Lilly and Company.

Authorship. All named authors meet the International Committee of Medical Journal Editors (ICMJE) criteria for authorship for this article, take responsibility for the integrity of the work as a whole, and have given their approval for this version to be published.

Authorship Contributions. L.K., D.D., and J.M. participated as trial investigators and reviewed and edited the manuscript. J.I.C. and M.A.D. contributed to the study design, the statistical analyses, the interpretation of the research, and writing the statistical methods. J.T. contributed to the study design, medical oversight, interpretation of the research, and writing of the manuscript. N.C.S. was responsible for medical oversight of the trial after unblinding for primary outcome assessment and contributed to interpretation of the research and writing the manuscript. J.B-V. was responsible for medical oversight during the trial and contributed to the study design, the data analysis and interpretation of the research, and writing the manuscript. All authors approved the final manuscript to be published. J.B-V. is the guarantor of this work and, as such, had full access to all the data in the study and takes responsibility for the integrity of the data and the accuracy of the data analyses.

Prior Presentation. Parts of this study were presented in abstract form at the 79th Scientific Sessions of the American Diabetes Association, San Francisco, CA, 6-11 June 2019, at the 55th Annual Meeting of the European Association for the Study of Diabetes, Barcelona, Spain, 16-20 September 2019, at the 80th Scientific Sessions of the American Diabetes Association, Virtual 
Meeting, 12-16 June 2020, at the Association of Diabetes Care \& Education Specialists Virtual Conference, 13-16 August 2020, and at the 56th Annual Meeting of the European Association for the Study of Diabetes, Virtual Meeting, 22-25 September 2020.

Disclosures. Leslie Klaff is an employee of Rainier Clinical Research which has received research grants from Eli Lilly and Company, Novo Nordisk, Sanofi, Medtronics, Abbott Diabetes, and Roche Diagnostics. Junnosuke Miura received speaker fees from Abbott, Astellas, AstraZeneca, Eli Lilly and Company, LifeScan Japan, Novo Nordisk, MSD, Ono, Taisho Pharma, Terumo, Nipro, and Sanofi, and consultant fees from Abbott, AstraZeneca, and Terumo. Dominik Dahl received speaker fees and research grants from AstraZeneca, Eli Lilly and Company, Novo Nordisk, Böehringer, Bayer, and Berlin Chemie. Jang Ik Cho, Mary Anne Dellva, Nanette C Schloot, Janet Tobian and Juliana Bue-Valleskey are employees and shareholders of Eli Lilly and Company. Nanette C Schloot is a guest scientist at the Institute for Clinical Diabetology, German Diabetes Centre, Dusseldorf, Germany. No other potential conflicts of interest relevant to this article were reported.

Compliance with Ethics Guidelines. The study was conducted in accordance with the ethical principles of the Declaration of Helsinki and the International Conference on Harmonisation Guideline for Good Clinical Practice. The PRONTO-T1D study protocol was reviewed and approved by institutional ethics committees at each study center. All participants provided written informed consent.

Data Availability. The datasets generated during and/or analyzed during the current study are available from the corresponding author on reasonable request.

Open Access. This article is licensed under a Creative Commons Attribution-NonCommercial 4.0 International License, which permits any non-commercial use, sharing, adaptation, distribution and reproduction in any medium or format, as long as you give appropriate credit to the original author(s) and the source, provide a link to the Creative Commons licence, and indicate if changes were made. The images or other third party material in this article are included in the article's Creative Commons licence, unless indicated otherwise in a credit line to the material. If material is not included in the article's Creative Commons licence and your intended use is not permitted by statutory regulation or exceeds the permitted use, you will need to obtain permission directly from the copyright holder. To view a copy of this licence, visit http://creativecommons.org/licenses/by$\mathrm{nc} / 4.0 /$.

\section{REFERENCES}

1. Heise T, Linnebjerg H, Coutant D, et al. Ultrarapid lispro lowers postprandial glucose and more closely matches normal physiological glucose response compared to other rapid insulin analogues: a phase 1 randomized, crossover study. Diabetes Obes Metab. 2020;22(10):1789-98.

2. Michael MD, Zhang C, Siesky AM, et al. Exploration of the mechanism of accelerated absorption for a novel insulin lispro formulation. Diabetes J. 2017;66(Suppl 1):968-P.

3. Pratt E, Leohr J, Heilmann C, Johnson J, Landschulz W. Treprostinil causes local vasodilation, is well tolerated, and results in faster absorption of insulin lispro. Diabetes. 2017;66(Suppl 1):975-P.

4. Leohr J, Dellva MA, Coutant D, et al. Ultra rapid lispro (URLi) accelerates insulin lispro absorption and insulin action vs. Humalog ${ }^{\circledR}$ (lispro) in patients with T2D. Diabetes. 2019;68(Suppl 1):1100-P.

5. Klaff L, Cao D, Dellva MA, et al. Ultra rapid lispro improves postprandial glucose control compared with lispro in patients with type 1 diabetes: results from the 26-week PRONTO-T1D study. Diabetes Obes Metab. 2020;22(10):1799-807.

6. Blevins T, Zhang Q, Frias JP, Jinnouchi H, Chang AM. Randomized double-blind clinical trial comparing ultra rapid lispro with lispro in a basal-bolus regimen in patients with type 2 diabetes: PRONTOT2D. Diabetes Care. 2020;43(12):2991-8.

7. Malecki MT, Cao D, Liu R, et al. Ultra-rapid lispro improves postprandial glucose control and time in range in type 1 diabetes compared to lispro: 
PRONTO-T1D continuous glucose monitoring substudy. Diabetes Technol Ther. 2020;22(11):853-60.

8. Bailey TS, Grunberger G, Bode BW, et al. American Association of Clinical Endocrinologists and American College of Endocrinology 2016 outpatient glucose monitoring consensus statement. Endocr Pract. 2016;22:231-61.

9. Bergenstal RM, Lunt H, Franek E, et al. Randomized, double-blind clinical trial comparing basal insulin peglispro and insulin glargine, in combination with prandial insulin lispro, in patients with type 1 diabetes: IMAGINE 3. Diabetes Obes Metab. 2016;18: 1081-8.

10. Heller S, Buse J, Fisher M, et al. Insulin degludec, an ultra-longacting basal insulin, versus insulin glargine in basal-bolus treatment with mealtime insulin aspart in type 1 diabetes (BEGIN Basal-Bolus type 1): a phase 3 , randomised, open-label, treat-totarget non-inferiority trial. Lancet. 2012;379: 1489-97.

11. Linnebjerg $\mathrm{H}$, Zhang $\mathrm{Q}$, LaBell E, et al. Pharmacokinetics and glucodynamics of ultra rapid lispro (URLi) versus Humalog ${ }^{\circledR}$ (lispro) in younger adults and elderly patients with type 1 diabetes mellitus: a randomised controlled trial. Clin Pharmacokinet. 2020. https://doi.org/10.1007/s40262-020-00903-0.

12. Lantus [prescribing information]. Bridgewater NJ: Sanofi-Aventis; 2019. http://products.sanofi.us/ Lantus/Lantus.pdf. Accessed 25 June 2020.

13. FIASP $^{\circledR}$ [prescribing information]. Bagsvaerd, Denmark: Novo Nordisk; 2019. https://www.novo-pi. com/fiasp.pdf. Accessed 25 June 2020. 